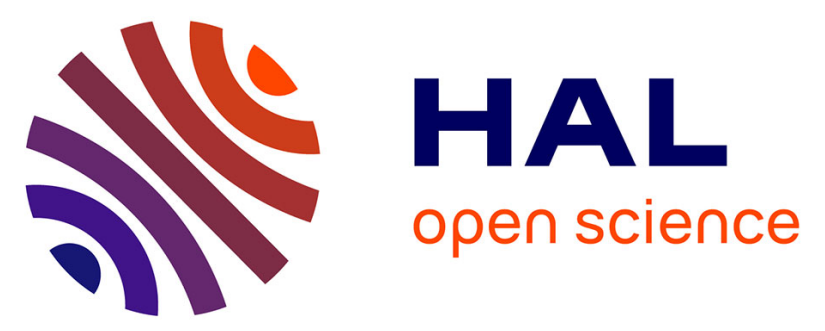

\title{
Considerations on Improving the Design of Cuff Electrode for ENG Recording - Geometrical Approach, Dedicated IC, Sensitivity and Noise Rejection
}

Fabien Soulier, Lionel Gouyet, Guy Cathébras, Serge Bernard, David Guiraud, Yves Bertrand

\section{To cite this version:}

Fabien Soulier, Lionel Gouyet, Guy Cathébras, Serge Bernard, David Guiraud, et al.. Considerations on Improving the Design of Cuff Electrode for ENG Recording - Geometrical Approach, Dedicated IC, Sensitivity and Noise Rejection. BIODEVICES: Biomedical Electronics and Devices, Jan 2008, Funchal, Madeira, Portugal. pp.180-185. lirmm-00334769

\section{HAL Id: lirmm-00334769 \\ https://hal-lirmm.ccsd.cnrs.fr/lirmm-00334769}

Submitted on 27 Oct 2008

HAL is a multi-disciplinary open access archive for the deposit and dissemination of scientific research documents, whether they are published or not. The documents may come from teaching and research institutions in France or abroad, or from public or private research centers.
L'archive ouverte pluridisciplinaire HAL, est destinée au dépôt et à la diffusion de documents scientifiques de niveau recherche, publiés ou non, émanant des établissements d'enseignement et de recherche français ou étrangers, des laboratoires publics ou privés. 


\title{
CONSIDERATIONS ON IMPROVING THE DESIGN OF CUFF ELECTRODE FOR ENG RECORDING Geometrical Approach, Dedicated IC, Sensitivity and Noise Rejection
}

\author{
Fabien Soulier, Lionel Gouyet, Guy Cathébras, Serge Bernard, \\ David Guiraud and Yves Bertrand \\ LIRMM, Université Montpellier II - CNRS - INRIA, 161 rue Ada, 34392 Montpellier, France \\ Firstname.Lastname@lirmm.fr
}

Keywords: Multipolar cuff electrode, regular tessellation, electroneurogram, action potential, Laplacian, selectivity, ASIC, multi-input differential amplifier.

\begin{abstract}
Cuff electrodes have several advantages for in situ recording ENG signal. They are easy to implant and not very invasive for the patient. Nevertheless, they are subject to background parasitic noise, especially the EMG generated by the muscles. We show that the use of cuff electrodes with large numbers of poles can to increase their sensitivity and their selectivity with respect to a efficient noise rejection. We investigate several configurations and compare the performances of a tripolar cuff electrode versus a multipolar one in numerical simulation.

One the other hand the use of cuff electrodes leads to the recording of the sum of the signals generated by all the axons within the nerve. This puts in evidence the need of signal separation techniques that require a great quantity of information. Again, we show that multipolar electrodes can solve this problem since poles can be switched one to another, provided that they are distributed along a regular tessellation.

Finally, we present the structure of an ASIC preamplifier processing a spatial filtering to obtain the Laplacian of the potential rejecting low-frequency noise.
\end{abstract}

\section{INTRODUCTION}

In a context of neural system pathologies such as spinal cord injury, Functional Electrical Stimulation (FES) techniques are the possible alternatives to restore lost sensory or motor abilities. These techniques consist in generating artificial contraction by electrical stimulation. In FES system a direct opened loop control doesn't allow efficient stimulation. In order to provide a loopback control we need sensory information (force, contact...) (Djilas et al., 2006). An attractive solution consists in using the natural sensors. The sensory information is propagated by associated afferent fibers. But unfortunately, in peripheral nerves the complete nerve activity due to the large number of axons makes the extraction of the studied signal particularly hard. Moreover the sensory signal seen through the nerve is a very low amplitude signal compared with the amplitude of parasitic signals. For instance, on a monopolar recording, EMG created by muscle activity have amplitude about three orders of magnitude higher than the ENG. In this context, the two main objectives to be able to exploit natural sensors are:

- to find a solution to separate the useful information from the complete ENG signal;

- to reject the parasitic external signals.

The classical solution consists in using multipolar electrodes, but from tripole (Ramachandran et al., 2005 ) to nine pole electrode (Winter et al., 2000; Taylor et al., 2004), the selectivity of the neural information is not efficient enough to be suitable in closed loop FES system. To achieve both a better sensitivity and efficient background noise rejection we propose a new configuration of the cuff electrode with a large number of poles regularly distributed onto the cuff. In this configuration, a group of poles can behave, with suitable low level analog signal processing, like a kind of a directive antenna. Moreover, the large number of poles will allow enough channels in order to apply source separation signal processing on the ENG. Of course, the directivity of the sensor relies on the 
quality of the subsequent low-level analog signal processing.

In this paper, we first show how to generalize the preprocessing operations on the recorded signal from tripolar to multipolar configuration using the Laplacian formalism. Then we discuss on the optimal pole placement around the nerve regarding tessellation methods. Both the electrode configuration and the associated preprocessing circuit result from this pole distribution and must be taken into account. We particularly focus on the hexagonal seven-pole electrode, presenting the associated seven input preamplifier and preliminary simulation results.

\section{EMG NOISE REJECTION}

Cuff electrodes have been the most used in the last ten years (Haugland et al., 1994; Jensen et al., 2002; Andreasen and Struijk, 2002). They are relatively easy to implant, they are not invasive for the nerve and implantation is very stable and thus allows chronic experiments. ENG can be recorded as the potential dif-

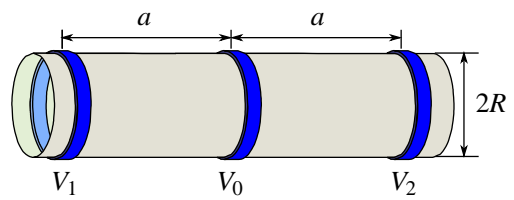

Figure 1: Tripolar electrode cuff model.

ference created on the electrodes by the charges associated to the action potentials (AP) propagating along the nerve fibers. Fig. 1 shows a typical tripolar cuff electrode. When recording with this kind of electrode, a classic method to reject parasitic signals consists in calculating the average of the potential differences between the central pole and each of the outer poles (Struijk and Thomsen, 1995; Pflaum et al., 1996):

$$
V_{\text {rec }}=\frac{\left(V_{0}-V_{1}\right)+\left(V_{0}-V_{2}\right)}{2}=V_{0}-\frac{V_{1}+V_{2}}{2}
$$

The last expression shows that this operation consists in:

1. averaging the signal on the outer poles, i.e. applying a low-pass spatial filter.

2. subtracting the result to the signal of the central pole, keeping only the high spatial frequencies.

Therefore, the recorded $V_{\text {rec }}$ signal can be considered as spatial high-pass filtered.

More precisely, this filter is a second-order one considering that the expressions

$$
\frac{1}{a}\left(V_{2}-V_{0}\right) \text { and } \frac{1}{a}\left(V_{0}-V_{1}\right)
$$

evaluate the first derivative $\frac{d V}{d x}$. Thus the difference

$\frac{1}{a}\left(\frac{\left(V_{2}-V_{0}\right)}{a}-\frac{\left(V_{0}-V_{1}\right)}{a}\right)=\frac{2}{a^{2}}\left(\frac{\left(V_{1}+V_{2}\right)}{2}-V_{0}\right)$

denotes the second derivative $\frac{d^{2} V}{d x^{2}}$ that is the onedimensional Laplacian of the potential. We can identify in the last expression the equation (1) without the known constant factor $-2 a^{-2}$.

Laplacian filters can reject both homogeneous potentials and linearly varying ones like those created by far EMG sources. The purpose of this new design is to build two-dimensional Laplacian using more poles to obtain isotropic rejection.

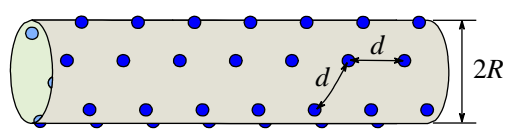

Figure 2: Multipolar electrode cuff model.

\section{POSITIONING THE POLES}

A tripolar cuff electrode (Demosthenous and Triantis, 2005) provides only one recording which is the superposition of all action potentials "seen" by the electrode at a given moment. The use of several poles on a cuff electrode (see Fig. 2) could allow us to record more signals, thus increase the quantity of neural data and facilitate the signal post-processing on the recording system.

In order to achieve optimal placement of poles, we must pay attention to three constraints:

1. The electrodes have to be placed all around the nerve, thus the poles have to be distributed onto the whole surface of the cuff.

2. The poles have to be equally spaced to simplify electronics in charge of analog signal preprocessing (weight coefficients in Laplacian preamplifier).

3. They have to be able to be substituted one to each other, so we take benefits of the maximum measurement locations, allowing powerful signal processing.

Since the cylindric shape of the cuff results from the wrapping of an initially plane device, these conditions imply to look for a regular tessellation of the plane as the positions of the poles or, more precisely, tessellations composed of regular polygons symmetrically tiling the plane. It is well known that there are exactly three type of regular tessellations (Weisstein, 
2002). They can be specified using the Schläfli symbols: $\{3,6\},\{4,4\}$ and $\{6,3\}$.

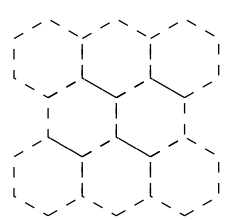

$\{6,3\}$

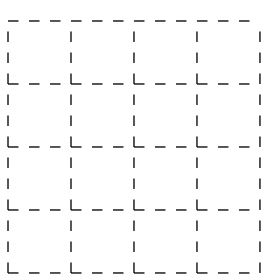

$\{4,4\}$

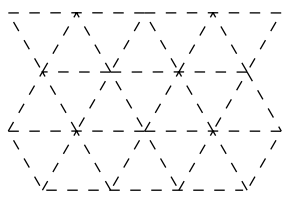

$\{3,6\}$
Figure 3: There are exactly three regular tessellations composed of regular polygons symmetrically tiling the plane.

The first symbol in the Schläfli notation denotes the shape of the patch (triangle, square or hexagon). On the figure 3 , each vertex corresponds to an pole. Each of them being surrounded by a number of equidistant poles given by the second Schläfli symbol, respectively 6,4 and 3 .

From the previous tessellations, one can build three kinds of electrodes by selecting one central pole and its closest neighbors. Namely, we can define a mesh of:

- triangular 4-pole electrodes,

- squared 5-pole electrodes,

- hexagonal 7-pole electrodes.

These candidates can be seen on the figure 4 and the resulting expressions for the Laplacian are:

$$
\begin{array}{ll}
V_{\text {rec }}=V_{0}-\frac{1}{3} \sum_{i=1}^{3} V_{i} & \text { for }\{6,3\} \\
V_{\text {rec }}=V_{0}-\frac{1}{4} \sum_{i=1}^{4} V_{i} & \text { for }\{4,4\} \\
V_{\text {rec }}=V_{0}-\frac{1}{6} \sum_{i=1}^{6} V_{i} & \text { for }\{3,6\}
\end{array}
$$

One can notice that the $\{4,4\}$ configuration correspond to the 2D Laplacian filter used in image processing (Gonzales and Woods, 1992).

\section{ENG AMPLIFIER}

Because of the very low level of processed signals we propose to perform the maximum of signal processing

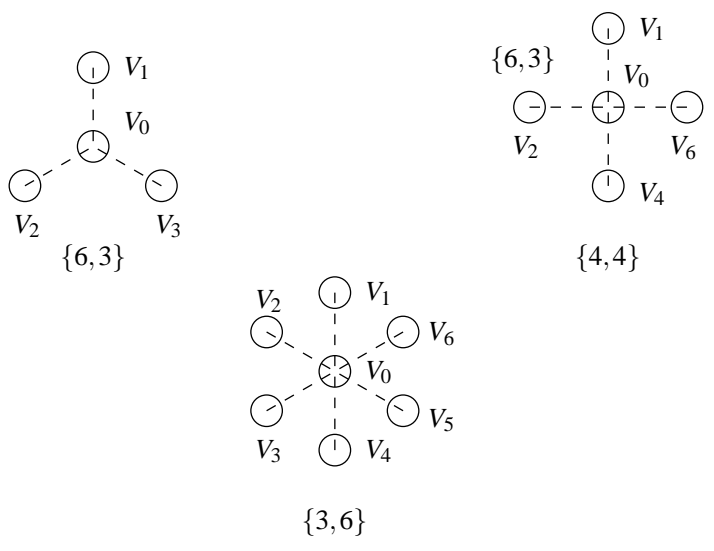

Figure 4: Three possible configurations of electrodes.

as close as possible to the nerve. The more complex operations to be considered are those with the hexagonal electrode.

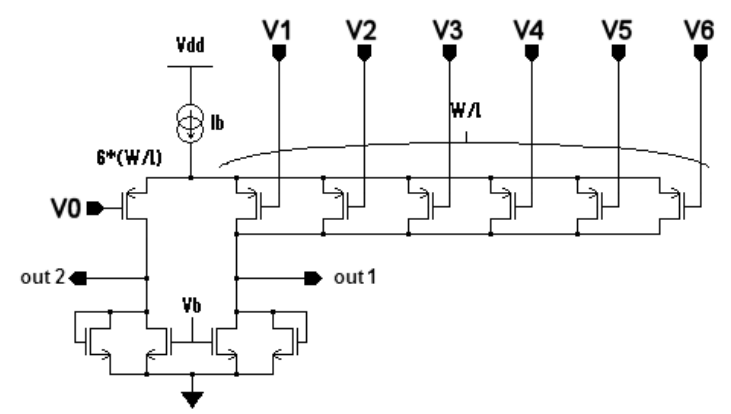

Figure 5: Seven input preamplifier.

For this purpose, we have designed a seven channels ASIC. Each channel compute a weighted difference between the measurement point and the six closest surrounding points. This is done in the analog domain using the preamplifier shown on figure 5. This preamplifier is build around a differential pair whose negative input transistor was split into six transistors (six times smaller, of course). It has a voltage gain that is about 100 and it is followed by an instrumentation amplifier whose gain is configurable between $6 \mathrm{~dB}$ and $80 \mathrm{~dB}$. Each channel is composed of one preamplifier followed by an instrumentation amplifier.

This circuit was designed to give an input-referred noise below $1 \mu \mathrm{V}_{\text {rms }}$, a CMMR above $60 \mathrm{~dB}$ and a sufficient gain, i.e greater than $60 \mathrm{~dB}$; all these parameters in the bandwidth of interest $(1 \mathrm{~Hz} \leq f \leq$ $3 \mathrm{kHz}$ ). The performances expected for this amplifier are given in Table 1 (the noise is measured in the band $1 \mathrm{~Hz}-3 \mathrm{kHz}$ ).

A microphotography of the fabricated circuit is 
Table 1: Amplifier characteristics (simulation).

\begin{tabular}{|l|c|}
\hline Active area (7 channels) & $1.16 \mathrm{~mm}^{2}$ \\
\hline Supply voltage & $3.3 \mathrm{~V}$ \\
\hline DC Current (Preamp) & $20 \mu \mathrm{A}$ \\
\hline Voltage gain (Preamp) & $100(40 \mathrm{~dB})$ \\
\hline CMRR (Preamp) & $80 \mathrm{~dB}(10 \mathrm{kHz})$ \\
\hline Voltage gain (Inst amp) & $2 \leq G \leq 10000$ \\
\hline CMRR (Full amp) & $80 \mathrm{~dB}(10 \mathrm{kHz})$ \\
\hline Input-ref. noise (Preamp) & $0.672 \mu \mathrm{V} \mathrm{RMS}$ \\
\hline Input-ref. noise (Full amp) & $0.677 \mu \mathrm{V} \mathrm{RMS}$ \\
\hline Bandwidth (Full amp) & $76 \mathrm{kHz}$ \\
\hline
\end{tabular}

presented Fig. 6. This circuit was designed in CMOS AMS $0.35-\mu \mathrm{m}$ technology.

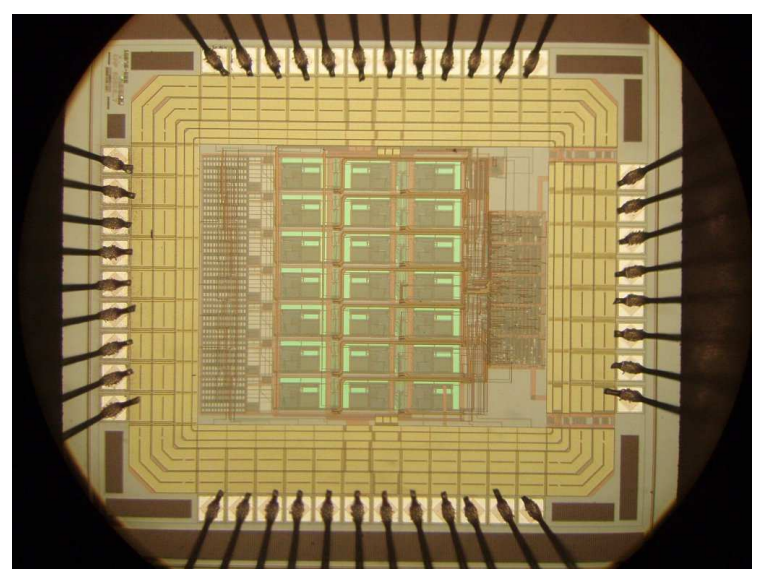

Figure 6: Microphotograph of the seven-channel prototype.

\section{NUMERICAL RESULTS}

\subsection{Action Potential modeling}

In order to evaluate the performances of multipolar electrodes, we need a model for the extracellular electric field created by an action potential. Let us consider a $10 \mu \mathrm{m}$ diameter myelinated axon. Its Ranvier nodes are $1 \mu \mathrm{m}$ long, while their diameter is $6 \mu \mathrm{m}$ and their spacing is $1 \mathrm{~mm}$. Let us call $\Omega$ the center of the Ranvier node. When the AP is present at this node, we can model it as a $6 \mu \mathrm{m}$ diameter circle, perpendicular to the axon axis, with a positive charge $+q$ at its center $(\Omega)$ and a negative charge $-q$ spread on the circle. The potential created at a point $M$ of the space by this AP can be approximated by:

$$
V(M)=\frac{q a^{2}}{8 \pi \varepsilon_{0} \varepsilon_{r} r^{3}}\left(1-\frac{3}{2} \sin ^{2} \psi\right)
$$

In this expression, $a$ is the radius of the Ranvier node ( $3 \mu \mathrm{m}), r$ is the distance between $\Omega$ and $M$, while $\psi$ is the angle between the axe of the axon and $\overrightarrow{\Omega M}$. This approximation, valid for $r \gg a$, is in good accordance with measurements. In particular, we can see that $V(M)$ is negative for $\psi=\pi / 2$ (Stein, 1980, page 81$)$. Last, $q$ can be easily estimated from the characteristics of the Ranvier node. For this study, we took $q \simeq 20 \mathrm{fC}$ and $\varepsilon_{r} \simeq 80$.

The model given by equation 7 was used to evaluate the sensitivity of the electrodes to action potentials occurring inside the nerve. For the evaluation of the rejection of parasitic signals, we must first recall that EMG are also action potentials, creating the same kind of electric field. But, in this case, we cannot make any assumption on the value of $\psi$. So, to evaluate the external sensitivity of electrodes, we chosen to use only a $1 / r^{3}$ model, unable to give voltages, but sufficient to compare the sensitivities of various electrodes.

We have limited the numerical study to the comparison of the classical tripolar cuff with the heptapolar (hexagonal shape $\{3,6\}$ ) electrode. We have also studied the effect of the wrapping to the performance of the heptapolar electrode: we consider a plane electrode and then a cylinder-wrapped one.

Given the position of a single AP we can easily calculate the induced potential on each pole of the cuff, since they are very small. For the tripolar cuff, we need to average the potential on each ring. This lead to an elliptic integral we have solved using numerical methods.

\subsection{Tripolar and heptapolar electrodes models}

In the following, we compare a tripolar cuff electrode, whose diameter is $2 R=3 \mathrm{~mm}$ and ring spacing is $a=4 R$, with one patch of the hexagonal cuff. To get comparable results, this hexagonal cuff has the same diameter $(2 R=3 \mathrm{~mm})$ and the spacing between poles is $d=R$. Since this patch is partially wrapped around the nerve, we considered also another patch perfectly flat.

For all the calculations, the coordinates were fixed as follow: the origin $O$ is at the center of the cuff electrode. The $O x$ axis is the axis of the nerve (and, obviously, of the cuff). The $O y$ axis passes by the center of the considered patch (which is perpendicular to this axe). Last the $O z$ axe is placed to form a direct trihedron with $O x$ and $O y$. 


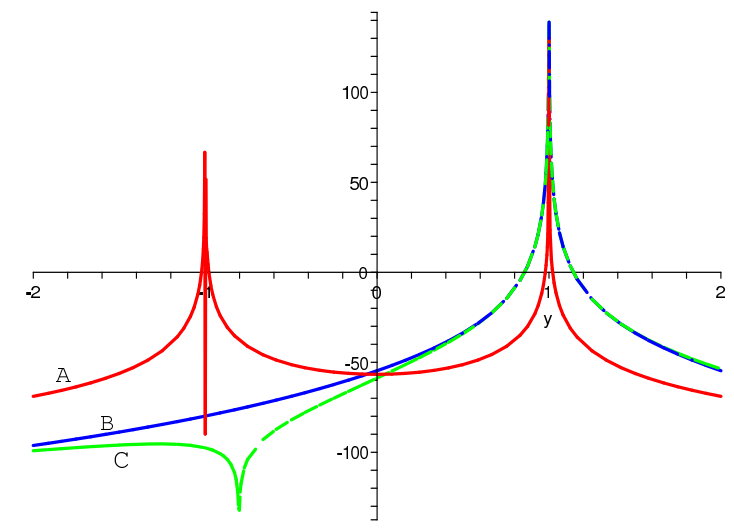

Figure 7: Radial sensitivities of (A) a tripolar cuff electrode, (B) a planar hexagonal patch and (C) a bent hexagonal patch. The vertical axis is in $\mathrm{dB} \mu \mathrm{V}$ and the unit for the horizontal axis is the radius $R$ of the electrode.

\subsection{Internal sensitivity}

Figure 7 shows the radial sensitivities of the three electrodes (tripolar cuff, planar hexagonal patch and wrapped hexagonal patch) that we compare. The vertical axis is the value of $V_{\text {rec }}($ in $\mathrm{dB} \mu \mathrm{V})$ calculated for an AP placed on the $O y$ axis, at abscissa $y R$. The graph shows clearly that while the sensitivity of the tripolar cuff is quasi constant on the section of the nerve, the sensitivity of the hexagonal patch is far higher (up to $30 \mathrm{~dB}$ ) when considering an AP located between the center of the patch and the center of the cuff.

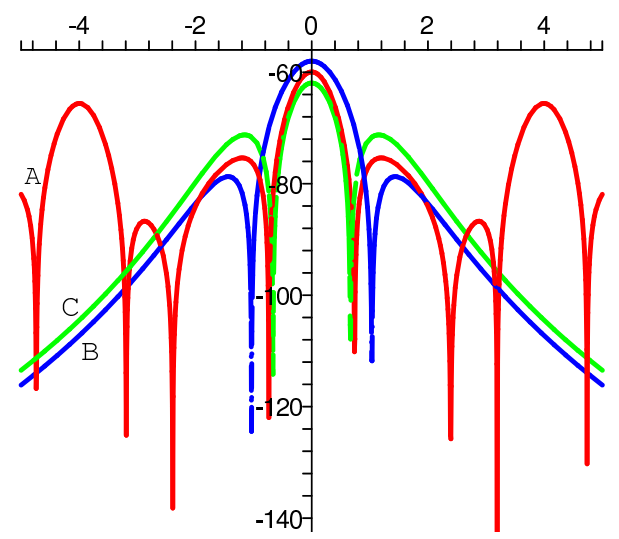

Figure 8: Longitudinal sensitivities on the axe of (A) a tripolar cuff electrode, (B) a planar hexagonal patch and (C) a bent hexagonal patch. The vertical axis is in $\mathrm{dB} \mu \mathrm{V}$ and the unit for the horizontal axis is the radius $R$ of the electrode.

Figures 8 and 9 show the longitudinal sensitivities of the three considered electrodes. On figure 8 , the sensitivity is computed for an AP placed on the $O x$

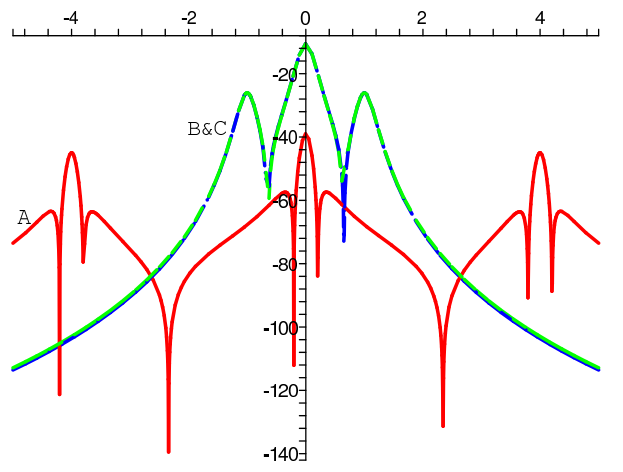

Figure 9: Longitudinal sensitivities on an off-center $(80 \%$ of R) axis of (A) a tripolar cuff electrode, (B) a planar hexagonal patch and $(\mathrm{C})$ a bent hexagonal patch. The vertical axis is in $\mathrm{dB} \mu \mathrm{V}$ and the unit for the horizontal axis is the radius $R$ of the electrode.

axe, while, on figure 9, the AP is placed on a line, parallel to $O x$, cutting $O y$ at abscissa $0.8 R$. On this later figure, we can see an increase of sensitivity of the tripolar cuff in the vicinity of the rings, but this remains far lower than the sensitivity of any of the hexagonal patches.

\subsection{External sensitivity}

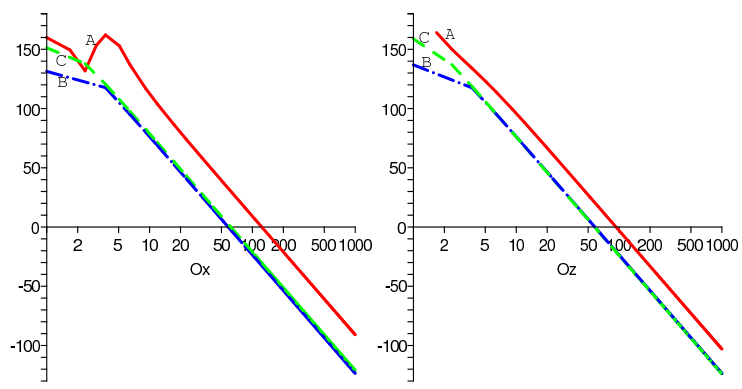

Figure 10: External relative sensitivity along $O x$ and $O z$ axes for (A) a tripolar cuff electrode, (B) a planar hexagonal patch and (C) a bent hexagonal patch. The vertical axis is in $\mathrm{dB}$ and the unit for the horizontal axis is the radius $R$ of the electrode.

The figure 10 show the external sensitivities of our three electrodes for an AP placed on the $O x$ or on the $O z$ axis of the electrode. As stated above, the quantity plotted is not a voltage, but is homogeneous to the reciprocal of the cube of a distance. Nevertheless, we can see on these two graphs that the hexagonal patches exhibit a better rejection of parasitic signals than the tripolar cuff. This improvement is of $32 \mathrm{~dB}$ for $O x$ and $20 \mathrm{~dB}$ for $O z$.

The same study conducted along the $O y$ axis (figure 11) shows that, while the planar patch continues 


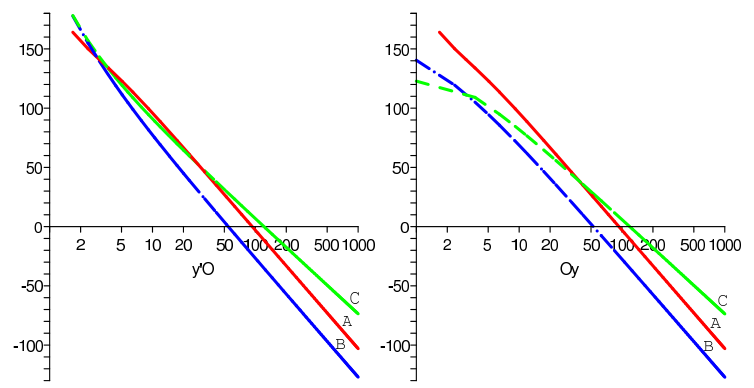

Figure 11: External relative sensitivity along the two halves of the $O y$ axis for (A) a tripolar cuff electrode, (B) a planar hexagonal patch and (C) a bent hexagonal patch. The vertical axis is in $\mathrm{dB}$ and the unit for the horizontal axis is the radius $R$ of the electrode.

to have the better rejection of parasitic signals, the wrapped hexagonal patch has a sensitivity decreasing slowly along this $O y$ axis. In fact, the bent hexagonal patch only begins to have larger sensitivity than the tripolar cuff for action potentials placed at more than fifty times the radius of the cuff, corresponding to approximately $7 \mathrm{~cm}$. At this distance, the parasitic signal could be neglected in comparison to ENG signal.

\section{CONCLUSION AND PERSPECTIVES}

We have presented here a method to build multipolar cuff electrodes and how to extract useful informations from the multiple channels. Although numerical investigations are still necessary to an exhaustive comparison of multipolar structures, the comparison between the classical tripolar cuff electrode for ENG recording and a multipolar electrode has shown that this new type of the design is very promising. In every simulation, multipolar electrodes prove to be more sensitive to sources located inside the nerve, and in almost every case they show better far source rejection.

We hope the improvement of the recorded signal given by this new design will allow the use of signal processing techniques such as source separation. Then, multipolar configurations could make it possible to estimate more precise parameters like the speed and the direction of propagation of the AP (Taylor et al., 2004; Rieger et al., 2006).

\section{REFERENCES}

Andreasen, L. N. S. and Struijk, J. J. (2002). Signal strength versus cuff length in nerve cuff electrode recordings.
49(9):1045-1050.

Demosthenous, A. and Triantis, I. F. (2005). An adaptive ENG amplifier for tripolar cuff electrodes. 40(2):412421.

Djilas, M., Azevedo Coste, C., Yoshida, K., and Cathébras, G. (2006). Interpretation of ENG signal for FES closed-loop control. In IFESS'06: 11th Annual Conference of the International Functional Electrical Stimulation Society, pages 85-87, Miyagi-Zao, Japan.

Gonzales, R. C. and Woods, R. E. (1992). Digital Image Processing. Addison-Wesley.

Haugland, M. K., Hoffer, J. A., and Sinkjaer, T. (1994). Skin contact force information in sensory nerve signals recorded by implanted cuff electrodes. IEEE Transactions on Rehabilitation Engineering, 2(1):1828.

Jensen, W., Sinkjaer, T., and Sepulveda, F. (2002). Improving signal reliability for on-line joint angle estimation from nerve cuff recordings of muscle afferents. IEEE Transactions on Neural Systems and Rehabilitation Engineering, 10(3):133-139.

Pflaum, C., Riso, R. R., and Wiesspeiner, G. (1996). Performance of alternative amplifier configurations for tripolar nerve cuff recorded ENG. In Engineering in Medicine and Biology Society, 1996. Bridging Disciplines for Biomedicine. Proceedings of the 18th Annual International Conference of the IEEE, volume 1, pages 375-376, Amsterdam.

Ramachandran, A., Sacristan, J., Lago, N., Dörge, T., Osès, M., Koch, K., and Hoffmann, K. (2005). Multipolar cuff electrodes with integrated pre-amplifier \& filter to interface peripheral nerves for FES application. In 10th Annual Conference of the International FES Society, Montreal, Canada.

Rieger, R., Schuettler, M., Pal, D., Clarke, C., Langlois, P., Taylor, J., and Donaldson, N. (2006). Very lownoise ENG amplifier system using CMOS technology. IEEE Transactions on Neural Systems and Rehabilitation Engineering, 14(4):427-437.

Stein, R. B. (1980). Nerve and Muscle. Plenum Press.

Struijk, J. J. and Thomsen, M. (1995). Tripolar nerve cuff recording: stimulus artifact, EMG and the recorded nerve signal. In Engineering in Medicine and Biology Society, 1995. IEEE 17th Annual Conference, volume 2, pages 1105-1106, Montreal, Que.

Taylor, J., Donaldson, N., and Winter, J. (2004). Multipleelectrode nerve cuffs for low-velocity and velocityselective neural recording. Medical and Biological Engineering and Computing, 42(5):634-643.

Weisstein, E. W. (2002). Tessellation. From MathWorldA Wolfram Web Resource. http://mathworld. wolfram.com/Tessellation.html.

Winter, J., Rahal, M., Taylor, N., Donaldson, N., and Struijk, J. (2000). Improved spatial filtering of ENG signals using a multielectrode nerve cuff. In 5th Annual Conference of the International Functional Electrical Stimulation Society, Aalborg, Denmark. 